\title{
Authentication of Golden-Berry P. ginseng Cultivar 'Gumpoong' from a Landrace 'Hwangsook' Based on Pooling Method Using Chloroplast-Derived Markers
}

\author{
Ho Jun Joh', Nam-Hoon Kim', Murukarthick Jayakodi', Woojong Jang ${ }^{1}$, Jee Young Park, Young Chang Kim², \\ Jun-Gyo $\mathrm{In}^{3}$, Tae-Jin Yang ${ }^{1,4} *$ \\ ${ }^{1}$ Deptartment of Plant Science, Plant Genomics and Breeding Institute, and Research Institute of Agriculture and Life Sciences, \\ College of Agriculture and Life Sciences, Seoul National University, Seoul 08826, Korea \\ ${ }^{2}$ Ginseng Research Division, National Institution of Horticultural and Herbal Science, Rural Development Administration, Eumseong \\ 27709, Korea \\ ${ }^{3}$ Laboratory of Resources and Analysis, R\&D Headquarters, Korea Ginseng Corporation, Daejeon 34337, Korea \\ ${ }^{4}$ Crop Biotechnology Institute/GreenBio Science and Technology, Seoul National University, Pyeongchang 25354, Korea
}

\begin{abstract}
Most ginseng cultivars bear red berry and only one cultivar 'Gumpoong' (GU) bears golden berry. GU is an elite cultivar bred by pedigree selection from a golden berry landrace (a mixed population) 'Hwangsook' (HS). We developed three unique polymorphic markers from complete chloroplast genome sequences of GU and HS. A population of GU showed uniform band amplicon against three chloroplast markers whereas HS population displayed mixed genotypes for both GU and HS. Using the characteristics of mixed genotypes in HS population, we developed a convenient method to differentiate GU and HS population by application of pooled DNA template for PCR analysis (pooling method). The pooling method revealed that the GU pool was identical with GU genotype while the HS pool showed both GU and HS genotype. The pooling method is a cost and time effective method for accurate authentication of both golden berry ginseng cultivars. The method is useful to protect GU products from its tentative counterfeits from seeds to mature plant stages as well as processed root products.
\end{abstract}

Keywords Panax ginseng, Chloroplast marker, Golden-berry, Pooling method, Authentication

\section{INTRODUCTION}

Ginseng (Panax ginseng) is a perennial herbal plant belonging to the Araliaceae family and widely known for its positive therapeutic effects on brain function, immune system, sexual function, atherosclerotic heart disease, diabetes, and cancer (Nam 2002; Choi 2008). Ginseng breeding is difficult and demands long time efforts for developing an elite cultivar because ginseng produce small number of seeds after four years of growth from seeds (Kwon et al. 2001). In Korea, more than 17 elite cultivars were developed by pedigree breeding through selection of a plant with good characteristics from three local landrace populations, Jakyung, Chungkyung, and Hwangsook (HS). Each of the local landrace is differentiated and grouped by the clear morphological characteristics including stem colors, berry colors; red stem Jakyung, green stem Chungkyung, and gold berry HS (Fig. 1). However, each landrace is genetically mixed population except uniform morphological traits of stem and berry colors.

The most elite cultivars were bred by identification of individual plant from farmer's field and inbreeding as

Received February 1, 2017; Revised February 7 2017; Accepted February 8, 2017; Published March 1, 2017

*Comesponding author Tae-Jin Yang, tjyang@snu.ac.kr, Tel: +82-2-880-4547, Fax: +82-2-873-2056 

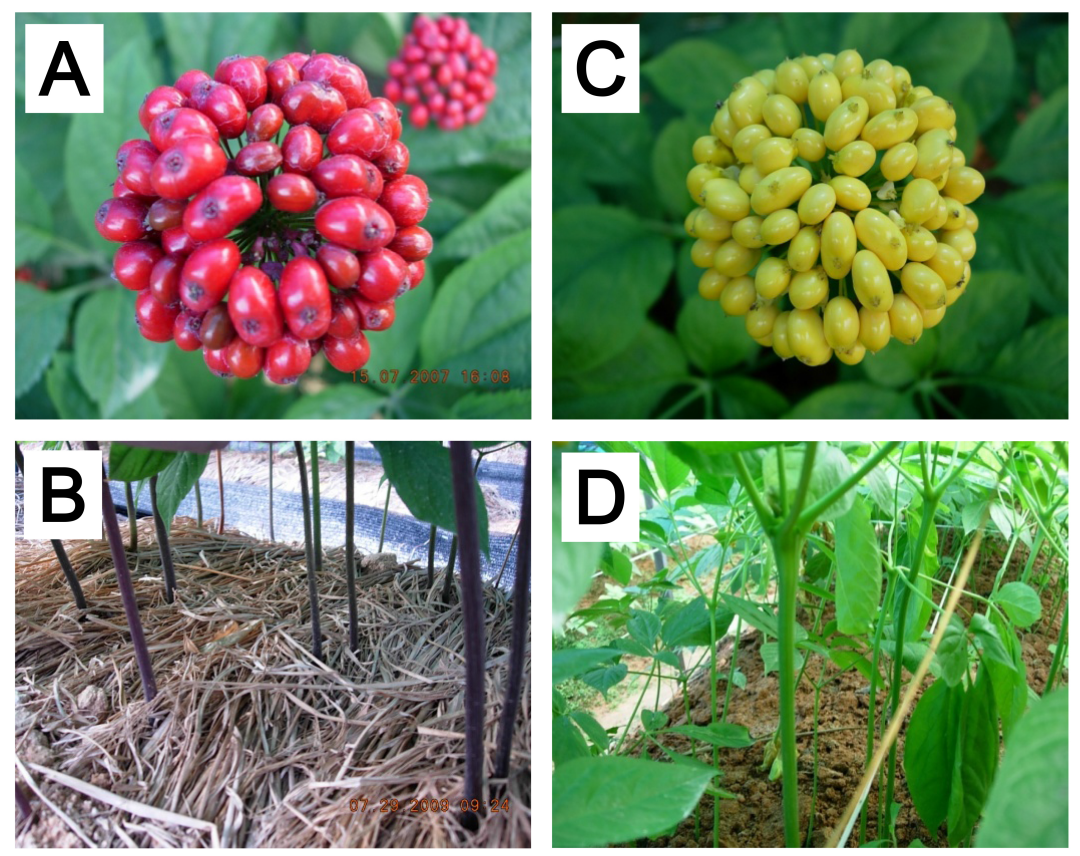

Fig. 1. Distinct characteristics of berry and stem of two $P$. ginseng landraces. (A, B) Red berry and violet stem of $P$. ginseng landrace Jakyung. (C, D) Golden berry and green stem of $P$. ginseng landrace Hwangsook (HS). Most of registered $P$. ginseng cultivars have developed from Jakyung except for $P$. ginseng cultivars Gumpoong (GU) developed from HS. GU shows very similar morphologies including berry color to HS.

pedigree in Korea (Kwon et al. 1998, 2001, 2003). Previously, the authentication of $P$. ginseng cultivars was relied on morphological differences of fully grown roots or aerial parts during cultivation. The environmental and developmental factors cause minor alterations in the morphological characteristics that may ultimately lead to differentiate cultivars wrongfully within large quantities of the samples (Wang et al. 2010). Molecular markers have been widely used for various applications including physical and genetic mapping of genome, identifying traits, genetic diversity and evolutionary analysis (Kumar et al. 2009). In ginseng, very few studies have used molecular markers such as RAPD (In et al. 2005), AFLP (Ha et al. 2002), SSR (Choi et al. 2011; Kim et al. 2012) and SNP (Wang et al. 2010; Sun et al. 2011) for differentiating ginseng species and cultivars.

Most of the ginseng landraces have reddish color berry (red berry) except HS, which has golden color berry (golden berry). Therefore, HS is easily distinguished from other $P$. ginseng cultivars. HS is a mixed population with various genotypes except uniform gold berry characteristics.
The Gumpoong (GU) cultivar has been developed by pedigree selection from superior individual of landrace HS. Hence, GU and HS have many similar morphological characteristics such as green stem and golden berry (RDA 2014). Nevertheless, GU possesses better root features such as a strong resistance to rusty roots than HS (Lee et al. 2015), which made the value of GU seeds almost three times higher than HS in the seed market. Consequently, the false trade of HS seed into GU seed became a major issue that disturbs ginseng market. Therefore, it is important to develop a fast and accurate method to distinguish this cultivar from HS landrace for ginseng industry.

Chloroplast genome has been used to investigate interand intra-species genetic relationship of plants (Kim et al. 2013; Jung et al. 2014). Previously, the complete chloroplast and ribosomal DNAs of $11 P$. ginseng cultivars was made and polymorphic markers were developed based on chloroplast genome sequence diversity (Kim et al. 2015). Here, we used three markers to authenticate GU and HS with an accurate and efficient system that use pooled DNA in a population scale. 


\section{MATERIALS AND METHODS}

\section{Plant materials and DNA preparation}

$P$. ginseng cultivar GU and landrace HS were the main materials used in this study. Two populations of GU and HS were collected from Korea Ginseng Corporation (KGC) and National Institute of Horticultural and Herbal Science (NIHHS) in Eumsung of Rural Development Administration (RDA). A total of 32 GU plants, 18 plants from KGC (Nos. 1-18) and 14 plants (Nos. 19-32) from RDA and 32 HS plants, 20 plants (Nos. 1-20) from KGC and 12 plants (Nos. 21-32) from RDA were inspected for their genotyping. As authentication controls for marker analysis, we used same samples of GU and HS plants used for chloroplast genomes assembly in our previous study (Kim et al. 2015). Leaves of each individual plant were collected for DNA preparation. Each leaf samples was grinded separately using liquid nitrogen and its whole genomic DNA was extracted using modified DNA isolation protocol using cetyltrimethylammonium bromide method (Allen et al. 2006). The quality and quantity of extracted DNA were examined using NanoDrop 1000 spectrophotometer (Thermo Fisher Scientific Inc., U.S.A).

\section{Selection and development of DNA markers}

From our previous study (Kim et al. 2015) which discovered several nucleotide polymorphisms between chloroplast genome sequences of GU and HS, two markers including one SNP and one InDel marker pgcpd02 (forward, 5'-ATTTCGGGGACTCACAGAAGTAC-3'; reverse, 5'-AAAGCAATTTACGCGAAGGA-3') and pgycf1 (forward, 5'-GGTATTAGTCTGGATACGGCAAA-3'; reverse,
5'-TCGAAAAGAAGGGTCACAAGA-3') respectively was used. In addition, we newly designed a dCAPS primer pairs named pgcp_ccsA from $\operatorname{ccs} A$ gene region of chloroplast genome. The dCAPS primer was designed as 5'-CAATAATCCAACTGTTGAATCACC-3' using dCAPS finder 2.0 (http://helix.wustl.edu/dcaps/; Neff et al. 2002) and the complementary primer was designed as 5'-GCTATGCAGCTCTTCTATGTGGA-3' using the online tool Primer3 (http://bioinfo.ut.ee/primer3-0.4.0/; Untergasser et al. 2012), with the product size of $199 \mathrm{bp}$ for GU and 223 bp for HS after digested with restriction enzyme.

\section{PCR amplification and electrophoresis}

PCR amplification was proceeded in total $25 \mu \mathrm{L}$ of reaction volume containing the following: $20 \mathrm{ng}$ of DNA template, $10 \mu \mathrm{M}$ of primer set, $5 \mathrm{mM}$ of dNTP, and one unit Taq DNA polymerase (Vivagen, Seongnam, Korea). The amplification condition was proceeded as follows: 5 minutes at $94^{\circ} \mathrm{C}, 35$ cycles of $94^{\circ} \mathrm{C} 20$ seconds, $54^{\circ} \mathrm{C} 20$ seconds, and $72^{\circ} \mathrm{C} 20$ seconds and then $72^{\circ} \mathrm{C}$ for 7 minutes. PCR products of dCAPS primers, pgcpd02 and pgcp_ccsA, were digested for about 4 hours with corresponding restriction enzymes, $S c a$ I and $S m a \mathrm{I}$, respectively (Table 1). PCR product was then separated by gel electrophoresis with 3\% agarose gel for pgcpd02 and pgcp_ccsA makers and $1 \%$ agarose gel for pgycf1 marker. Gel was stained with ethidium bromide and visualized under UV lamps for manual genotyping.

\section{Pooling method}

To differentiate GU and HS efficiently on population level, we applied DNA pooling method by creating a DNA

Table 1. Polymorphic sites and marker information in chloroplast genomes of GU and HS.

\begin{tabular}{llccccc}
\hline \hline Type & Position & $\begin{array}{c}\text { Nucleotide position } \\
\text { GU / HS }\end{array}$ & $\begin{array}{c}\text { Polymorphisms } \\
\text { GU / HS }\end{array}$ & Primer ID & $\begin{array}{c}\text { Product size (bp) } \\
\text { GU/HS }\end{array}$ & $\begin{array}{c}\text { Restriction } \\
\text { enzyme }\end{array}$ \\
\hline InDel & $y c f 1$ & $111,305 / 111,304$ & $57 \times 4 / 57 \times 3$ & pgycf1 $^{\mathrm{z})}$ & $729 / 672$ \\
InDel & rps16 intron & 5,473 & $(\mathrm{C})_{9} /(\mathrm{C})_{8}$ & & & \\
SNP & rpoC2 & $21,345 / 21,344$ & $\mathrm{~T} / \mathrm{C}$ & pgcpd02 $^{\mathrm{z})}$ & $177 / 200$ & ScaI \\
SNP & $c c s A$ & $117,427 / 117,369$ & $\mathrm{G} / \mathrm{A}$ & pgcp_ccsA $^{\mathrm{y})}$ & $199 / 223$ & SmaI \\
SNP & $y c f 1$ & 127,069 & $\mathrm{~A} / \mathrm{T}$ & & & \\
\hline
\end{tabular}

Polymorphic sites and marker information were from Kim et al. (2015).

${ }^{\mathrm{z})}$ Developed in Kim et al. (2015).

${ }^{\mathrm{y})}$ Developed in this study. 
pool of GU and HS population for PCR analysis, as the concept of bulked analysis (Michelmore et al. 1991). PCR amplification and gel electrophoresis was processed using three markers pgcpd02, pgcp_ccsA and pgycf1 with the same procedure and condition as those for analysis of the individual samples. Gel was stained with ethidium bromide and visualized under UV lamps for manual genotyping.

\section{RESULTS}

\section{Sequence diversity of chloroplast genome between} GU and HS

Golden berry is distinct characteristics compare to red berry in ginseng. Most popular cultivating ginseng cultivars are red berry bearing cultivars (Fig. 1A) but GU is the

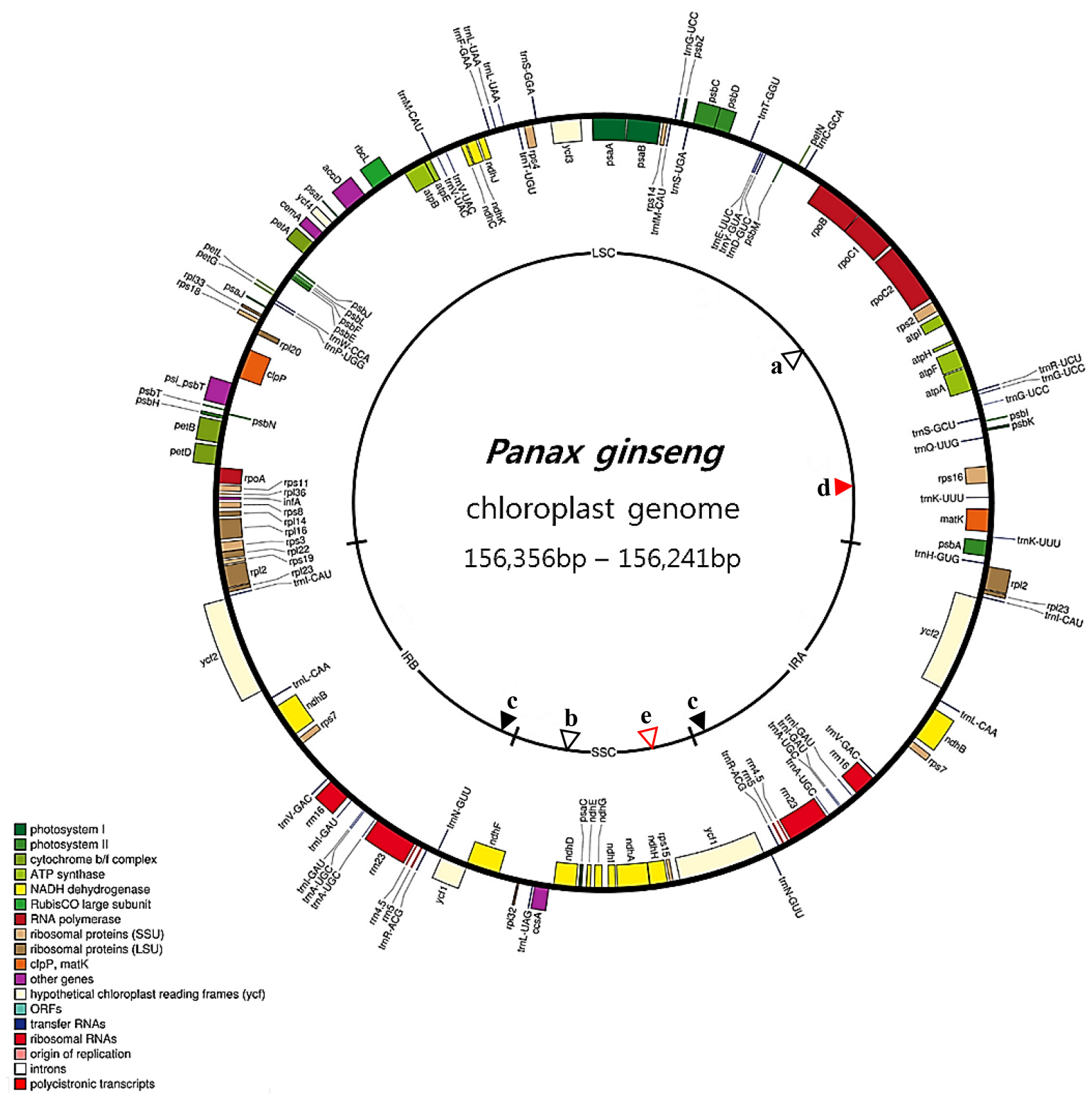

Fig. 2. Location of InDel and SNP variations between chloroplast genome sequences of GU and HS. The intra-species polymorphic sites were identified among 11 ginseng cultivars in our previous study (Kim et al. 2015), of which five (a-e) showed polymorphisms (two InDels as color-filled triangles and three SNPs as empty triangles) between GU and HS. Among the five polymorphism sites, one InDel and two SNPs (indicated by a, b and c) were used to design makers in this study. a, pgcpd02; b, pgcp_ccsA; c, pgycfl. pgcp_ccsA marker was newly developed in this study. Polymorphic site c was duplicated in two inverted repeats (IRA and IRB). 
only cultivar bearing golden berry (Fig. 1C). Our previous study found 12 intra-species polymorphisms (6 SNPs and 6 InDels) in complete chloroplast genomes among $11 P$. ginseng cultivars (Kim et al. 2015). Among those, five polymorphic sites (three SNPs and two InDels) were identified between chloroplast genomes of two gold berry ginseng cultivars, one inbred cultivar GU and one mixed landrace population HS. All five polymorphisms were identified in four genic regions of the chloroplast genomes (rpoC2, ccsA, ycfl and rps16) (Table 1, Fig. 2).

\section{Chloroplast genome marker derived from sequence divensity of GU and HS}

From the five polymorphic sites, three were chosen for marker designing and analysis. Two markers, pgycfl identifying copy number variation of $57 \mathrm{bp}$ tandem repeat (TR) in $y c f 1$ gene region and pgcpd02 dCAPS marker detecting the SNP in rpoC2 gene region, were chosen. In addition, we developed a new pgcp_ccsA dCAPS marker detecting SNP located in $\operatorname{ccs} A$ gene region (Fig. 3). We disregarded the remaining InDel from rps 16 and SNP from $y c f 1$ gene due to the difficulties in developing marker. Finally, three markers, pgcpd02, pgycf1 and pgcp_ccsA, were applied to $\mathrm{GU}$ and $\mathrm{HS}$ populations that were collected from two different ginseng-breeding fields as described in Material and Methods.

\section{Inspection of $\mathrm{GU}$ and $\mathrm{HS}$ populations}

All three markers successfully amplified their specific PCR amplicons for each individual in GU and HS population. Each individual plant could be genotyped as GU type or HS type for each of three markers (Fig. 4). All 32 individual plants in GU populations from both KGC and RDA populations showed the same genotype as that of control GU plant when applied all three markers. Meanwhile, 32 individual plants in HS population did not show identical genotypes but mixed genotypes for both $\mathrm{GU}$ and HS for all three markers (Table 2, Fig. 4).

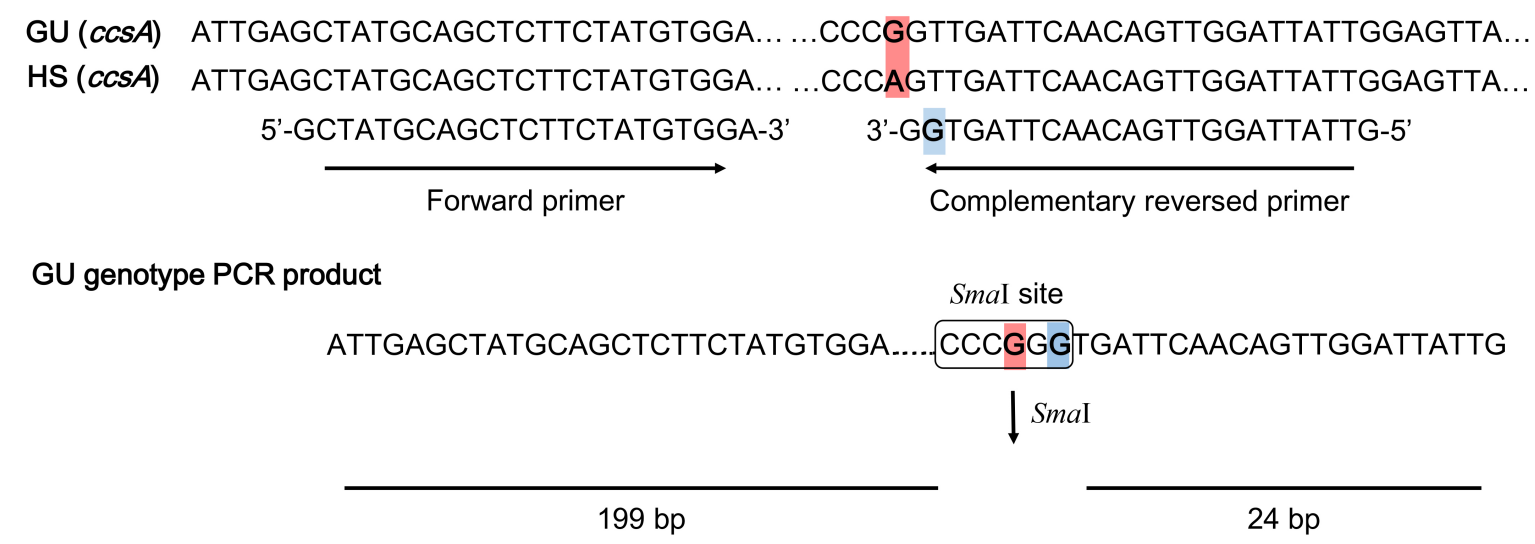

HS genotype PCR product

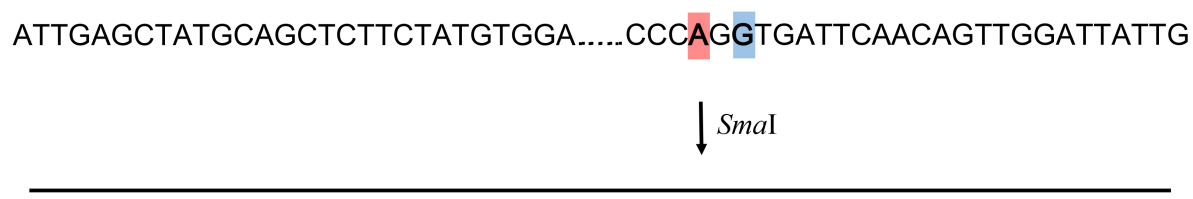

$223 \mathrm{bp}$

Fig. 3. Diagram of dCAPS marker pgcp_ccsA for discriminating GU and HS sequence. Nucleotide in red box represents single nucleotide polymorphism (SNP) in $\operatorname{ccs} A$ gene region between GU and HS. Nucleotide in blue box represent designed nucleotide mismatch for SmaI cutting. This dCAPS marker is developed to digest only GU sequence. After PCR amplification and SmaI cutting, PCR product of GU is cleaved into two fragments of 199 bp and 24 bp, while product of HS is not cleaved and remains to be $223 \mathrm{bp}$. These size differences can easily be detected through electrophoresis. 
A Individual plants in Gumpoong population

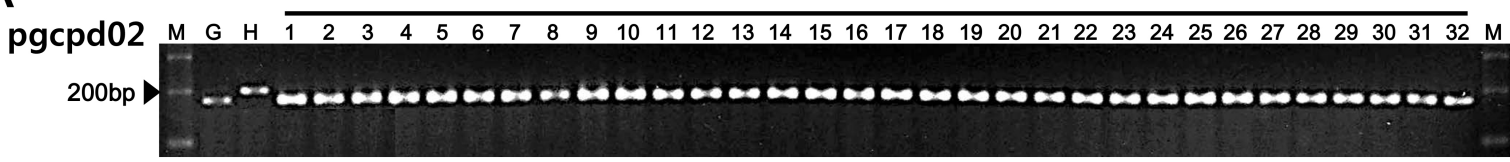

pgcp_ccsA
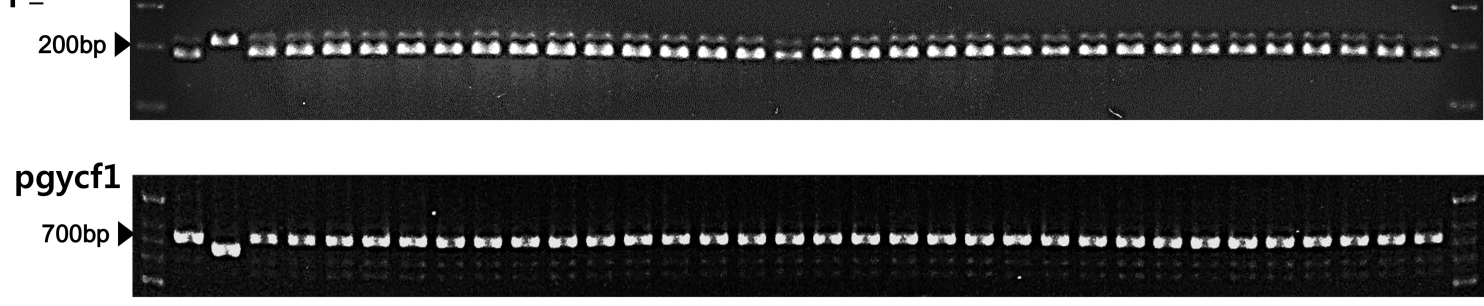

B Individual plants in Hwangsook population
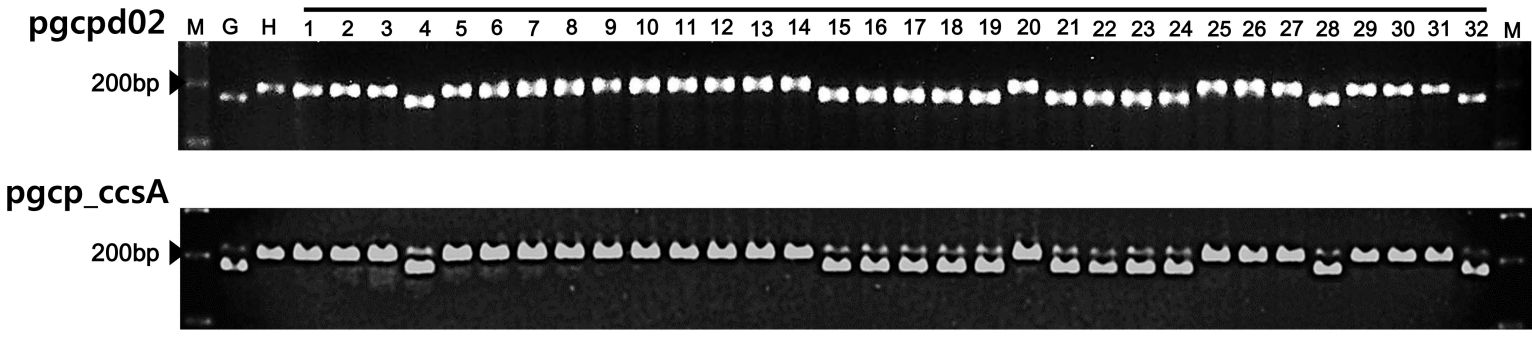

pgycf1

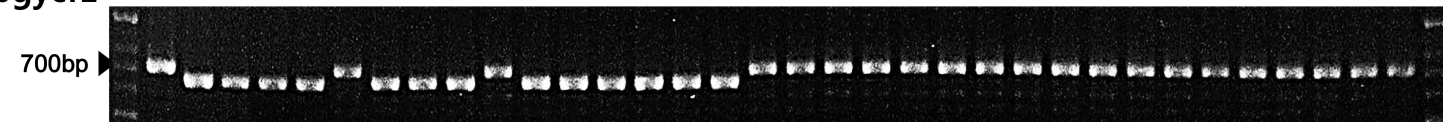

Fig. 4. Application of three molecular markers to individual plants in GU and HS population. A. Marker analyses of individual plants in GU population. B. Marker analyses of individual plants in HS population. Numbers (1-32) indicate plant nos. as described in Materials and Methods. G and $\mathrm{H}$ indicate authentication controls for GU and HS, respectively. Electrophoresis was performed using $1 \%$ agarose gel for pgcpd02 and pgcp_ccsA marker, and $3 \%$ agarose gel for pgycf1 marker. M represents 100-bp DNA ladder.

Table 2. The genotyping data of individual plant in GU and HS population using three markers.

\begin{tabular}{lcccccc}
\hline \hline \multirow{2}{*}{ Primer ID } & \multicolumn{2}{c}{ Gumpoong $(\mathrm{GU})$} & & \multicolumn{2}{c}{ Hwangsook $(\mathrm{HS})$} \\
\cline { 2 - 3 } \cline { 5 - 6 } & No. of GU type & No. of HS type & & No. of GU type & No. of HS type \\
\hline pgcpd02 & 32 & 0 & 0 & 12 & 20 \\
pgcp_ccsA & 32 & 0 & & 12 & 20 \\
pgycf1 & 32 & & 20 & 12 \\
\hline
\end{tabular}

The frequency of genotypes was different in two HS populations. For pgycf1 marker, two plants in KGC HS population (plant nos. 4 and 8 ) and all 18 plants in RDA HS population (plant nos. 15-32) showed GU genotype and the remaining 12 plants showed HS genotype (Fig. 4). For pgcpd02 and pgcp_ccsA marker, one plant in KGC HS (plant no. 4) and 11 plants in RDA HS (plant nos. 15-19,
21-24, and 28) were identified as GU genotype and the remaining 20 plants were identified as HS genotype (Fig. 4).

Pooling method to authenticate GU and HS population

Although our three DNA markers could discriminate genotype of each GU and HS individual clearly, it was obvious that large number of individuals in a population 

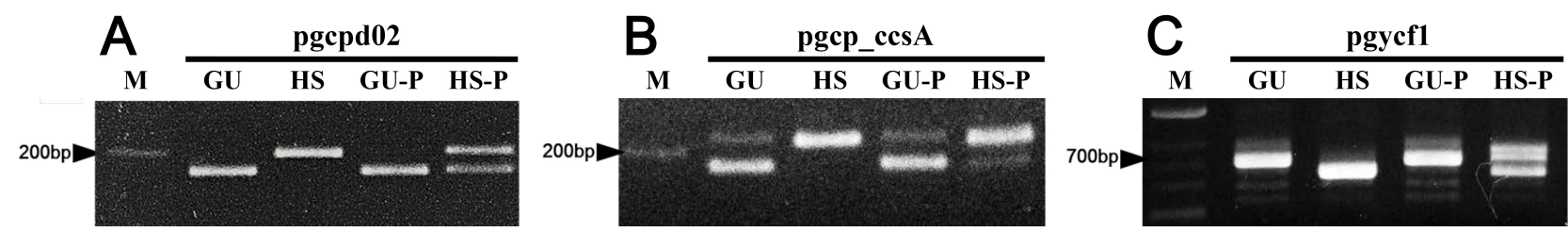

Fig. 5. Bulked population analysis of GU and HS with three molecular marker. GU and HS represent authentication controls, respectively. GU-P and HS-P represent the 32 plant bulked DNA template sample of GU and HS population, respectively. Electrophoresis was performed using $1 \%$ agarose gel for pgcpd02 and pgcp_ccsA marker, and 3\% agarose gel for pgycf1 marker. M represents 100-bp DNA ladder.

should be inspected to determine homogeneity of GU and HS population. Analysis of large number of plants is easy technically but it is high costs and time-consuming task. Thus, we proposed a pooling method to authenticate each population without PCR analysis of large numbers of plants. The genomic DNAs from GU and HS population plants were pooled, respectively, and the PCR amplification was performed using the pooled DNA as template with the three markers, pgcpd02, pgcp_ccsA, and pgycf1. GU pool (GU-P) displayed same and uniformed band pattern of amplicon as control GU sample, whereas HS pool (HS-P) displayed mixed band patterns of two types of GU and HS band (Fig. 5).

\section{DISCUSSION}

\section{The chloroplast genome-based markers between two golden-beny cultivars, GU and HS}

In history of $P$. ginseng cultivation, three landraces, Jakyung, Chungkyung, and Hwangsook, were efficiently discriminated using stem color or berry color such as Figure 1 (RDA 2014). Most of ginseng cultivars were developed from pedigree selection (RDA 2014) from population of three landraces and thus most of them exhibited similar phenotype with landraces. Although efforts have been made for differentiating cultivars in $P$. ginseng using molecular markers (Kim et al. 2013, 2015), the differentiation of landrace from cultivars remain elusive. Compared to other crop plants, the registered $P$. ginseng cultivars have low uniformity. Therefore, it is well appropriate to use chloroplast genome-based markers to distinguish each cultivar as they show diversity at both inter- and intra-species level.

As sequencing technology and bioinformatics tools have advanced, the studies on complete assembly of chloroplast genome and development of barcoding markers to differentiate the plant species or cultivars have also been increased. We identified 12 polymorphic markers from 11 ginseng cultivars (Kim et al. 2015). Although the three chloroplast genome-derived markers, pgcpd02, pgcp_ccsA, and pgycf1, showed clear polymorphism between GU and HS, these three markers showed common genotypes with some other cultivars among 11 ginseng cultivars. One ginseng cultivar 'Chunpoong' showed same genotype with HS for pgcpd02 and a cultivar 'Cheongsun' showed same genotypes with GU for two markers in rpoc2 and ccsA genes (Kim et al. 2015). GU and HS bearing golden berries can easily be differentiated from the rest of $P$. ginseng cultivars bearing red berry by morphology. On the other hand, it is difficult to differentiate GU and HS based on morphology, although the market value is highly different that has led delusion of seed market. Therefore, we concluded that it is appropriate to use these markers to evaluate only GU and HS.

\section{Evaluation of GU and HS population with three DNA markers}

The landraces are locally adapted plant population over centuries and have more genetic diversities than cultivars. Originally, the cultivar GU was bred by pureline selection of one superior individual of landrace HS and followed pedigree selection. Thus, some individuals in HS population may contain same genotype as GU such as shown by three markers in this study (Fig. 4). All of GU plants showed same genotype for three markers, indicating the 
GU has uniform genotype that make GU as registered cultivar. With such characteristic shared by landrace HS and GU, the uncertainty would arise when we use small number of plant samples and markers for authentication of GU and HS. We observed also that pgycf1 showed GU genotype for all of RDA HS population (Fig. 4. Plant nos. 21-32). Furthermore, 12 HS individual plants such as no. 4, 15-19, 21-24, 28, and 32 show GU genotypes for all three markers (Fig. 4) that would lead to wrong decision to distinguish of HS from GU. We suggest to use a couple of markers together against more than thirty samples per population to authenticate the golden berry bearing population. It is also expected that the differentiation accuracy would be improved with increasing number of plant samples and DNA markers.

\section{Pooling method to authenticate an elite cultivar from landrace with mixed genotypes}

Based on our results, we realized that the discrimination power is low when we use small number of plant samples. Applying markers onto large numbers of plants or population would require more cost and efforts. To utilize the molecular marker into efficient and high-throughput manner, we designed and used a pooling method. Each of three markers used in this study was capable of differentiating GU from HS population. Even though each marker could differentiate GU or HS pools, we strongly suggest to use all three markers for evaluating GU or HS population.

The general profile of plant authentication analysis relies more on morphological trait differences or molecular marker system among inbred cultivars. However, under the condition like differentiating cultivar and landrace, new concepts and analysis methods are necessary. In this study, we have evaluated molecular markers to efficiently differentiate cultivar GU from landrace HS by utilizing pooling method. This is the first study that focused on authentication of an elite ginseng cultivar from its original landrace mixed population at the population level. We believe that this method can be applied in discriminating other $P$. ginseng cultivars from landrace or between Panax species and other medicinal crops.

The pooling method used in this study is cheap, practical and exact to discriminate the mixed population. The authentication could be more precise by increasing individuals in each population without extra cost, which is a big advantage for practical application. The pooling method can also be applied to tissue pools derived from a population. Furthermore, the method can be applied to large number of seeds to evaluate the authentic seeds of GU cultivar and seed purity, collection of small piece of leaves derived from large numbers of plants in same field, as well as collection of piece of roots in a root bulk in the ginseng market or processing line of ginseng product industry.

\section{ACKNOWLEDGEMENTS}

This study was carried out with the support of "Cooperative Research Program for Agriculture Science \& Technology Development (Project Nos. PJ01103001)", Rural Development Administration, and a grant (16172MFDS229) from Ministry of Food and Drug Safety in 2016, Republic of Korea. We thank Dr. Sang-Choon Lee for critical comments.

\section{REFERENCES}

Allen GC, Flores-Vergara MA, Krasnyanski S, Kumar S, Thompson WF. 2006. A modified protocol for rapid DNA isolation from plant tissues using cetyltrimethylammonium bromide. Nat. Protoc. 1: 2320-2325.

Choi HI, Kim NH, Kim JH, Choi BS, Ahn IO, Lee JS, et al. 2011. Development of reproducible EST-derived SSR markers and assessment of genetic diversity in Panax ginseng cultivars and related species. J. Ginseng Res. 35: 399.

Choi KT. 2008. Botanical characteristics, pharmacological effects and medicinal components of Korean Panax ginseng C A Meyer. Acta. Pharm. Sinic. 29: 1109-1118.

Ha W, Shaw P, Liu J, Yau FC, Wang J. 2002. Authentication of Panax ginseng and Panax quinquefolius using amplified fragment length polymorphism (AFLP) and directed amplification of minisatellite region DNA (DAMD). J. Agric. Food. Chem. 50: 1871-1875.

In DS, Kim YC, Bang KH, Chung JW, Kim OT, Hyun DY, et 
al. 2005. Genetic relationships of Panax species by RAPD and ISSR analyses. Kor. J. Med. Crop Sci. 13: 249-253.

Jung J, Kim KH, Yang K, Bang KH, Yang TJ. 2014. Practical application of DNA markers for high-throughput authentication of Panax ginseng and Panax quinquefolius from commercial ginseng products. J. Ginseng Res. 38: 123-129.

Kim JH, Jung JY, Choi HI, Kim NH, Park JY, Lee Y, et al. 2013. Diversity and evolution of major Panax species revealed by scanning the entire chloroplast intergenic spacer sequences. Genet. Resour. Crop Evol. 60: 413-425.

Kim K, Lee SC, Lee J, Lee HO, Joh HJ, Kim NH, et al. 2015. Comprehensive survey of genetic diversity in chloroplast genomes and 45S nrDNAs within Panax ginseng species. PLoS One 10: e0117159.

Kim NH, Choi HI, Ahn IO, Yang TJ. 2012. EST-SSR marker sets for practical authentication of all nine registered ginseng cultivars in Korea. J. Ginseng. Res. 36: 298-307.

Kumar P, Gupta VK, Misra AK, Modi DR, Pandey BK. 2009. Potential of molecular markers in plant biotechnology. Plant Omics 2: 141-162.

Kwon WS, Chung CM, Kim YT, Lee MG, Choi KT. 1998. Breeding process and characteristics of KG101, a superior line of Panax ginseng CA Meyer. J. Ginseng Res. 22: 11-17.

Kwon W, Lee M, Lee J. 2001. Characteristics of flowering and fruiting in new varieties and lines of Panax ginseng CA Meyer. J. Ginseng Res. 25: 41-44.

Kwon WS, Lee JH, Park CS, Yang DC. 2003. Breeding process and characteristics of Gopoong, a new variety of Panax ginseng CA Meyer. J. Ginseng Res. 27: 86-91.

Lee JH, Lee JS, Kwon WS, Kang JY, Lee DY, In JG, et al. 2015. Characteristics of Korean ginseng varieties of Gumpoong, Sunun, Sunpoong, Sunone, Cheongsun, and Sunhyang. J. Ginseng Res. 39: 94-104.

Michelmore RW, Paran I, Kesseli RV. 1991. Identification of markers linked to disease-resistance genes by bulked segregant analysis: a rapid method to detect markers in specific genomic regions by using segregating populations. Proc. Natl. Acad. Sci. U. S A. 88: 9828-9832.

Nam KY. 2002. Clinical applications and efficacy of Korean ginseng. J. Ginseng Res. 26: 111-131.

Neff MM, Turk E, Kalishman M. 2002. Web-based primer design for single nucleotide polymorphism analysis. Trends Genet. 18: 613-615.

Rural Development administration (RDA). 2014. Korean ginseng-Agricultural technique guide 103. RDA. Korea

Sun H, Wang HT, Kwon WS, Kim YJ, In JG, Yang DC. 2011. A simple and rapid technique for the authentication of the ginseng cultivar, Yunpoong, using an SNP marker in a large sample of ginseng leaves. Gene 487: 75-79.

Untergasser A, Cutcutache I, Koressaar T, Ye J, Faircloth BC, Remm M, et al. 2012. Primer3 - new capabilities and interfaces. Nucleic Acids Research 40:e115.

Wang H, Sun H, Kwon WS, Jin H, Yang DC. 2010. A PCR-based SNP marker for specific authentication of Korean ginseng (Panax ginseng) cultivar "Chunpoong". Mol. Biol. Rep. 37: 1053-1057. 